\title{
Probiotics and diarrhea in children
}

Two studies published in Alimentary Pharmacology and Therapeutics provide further evidence for the beneficial effects of certain probiotic strains in reducing the incidence or duration of diarrhea in children.

In the first study, Szajewska and colleagues conducted a meta-analysis to investigate the beneficial effects of Lactobacillus rhamnosus GG for preventing health-care-associated diarrhea in children.

Nosocomial infections, particularly gastrointestinal infections, are common in children and can result in a prolonged hospital stay and increased medical costs. "There is evidence to suggest that specific probiotics may contribute to the prevention or treatment of diarrheal diseases," explains Szajewska. "However, there are also conflicting studies reporting no preventive effects of probiotics." Thus, the researchers decided to focus on the efficacy of administering $L$. rhamnosus GG to hospitalized children to prevent health-care-associated diarrhea.

Three randomized controlled trials, including a total of 1,092 children, were included in the meta-analysis. "The use of L. rhamnosus GG seems to be an effective strategy for preventing or reducing the risk of health-care-associated diarrhea, including that of rotavirus origin, in the pediatric setting," the authors report. "Although L. rhamnosus GG was unable to reduce the duration of hospital stay, other possible outcomes deriving from this intervention are possible, such as a reduction in costs related to medications, procedures or additional laboratory testing."

In the second study, Vandenplas et al. performed a prospective, randomized controlled trial to compare the synbiotic food supplement Probiotical ${ }^{\mathrm{TM}}$ (Laboratoires Phacobel, Belgium) with placebo to reduce the duration of acute diarrhea (gastroenteritis) in children. "Probiotics are used a lot in the treatment of gastroenteritis," says Vandenplas. "But it is recommended that every commercialized product should be tested because there are many poor quality products available on the market."

111 children with acute diarrhea were included in the trial. The children were randomly allocated to receive either placebo or the synbiotic (containing Streptoccoccus thermophilus, L. rhamnosus, L. acidophilus, Bifidobacterium lactis, B. infantis and fructo-oligosaccharides).
The median duration of diarrhea was significantly shorter in the group who received the synbiotic: 3 days in the synbiotic group versus 4 days in the group who received placebo $(P<0.005)$. Furthermore, less additional medication was administered in the synbiotic group, and physicians were more satisfied with this treatment compared with placebo.

The researchers plan to take this work forward by investigating the cost-saving effect of administering this product to children with acute gastroenteritis.

Isobel Franks

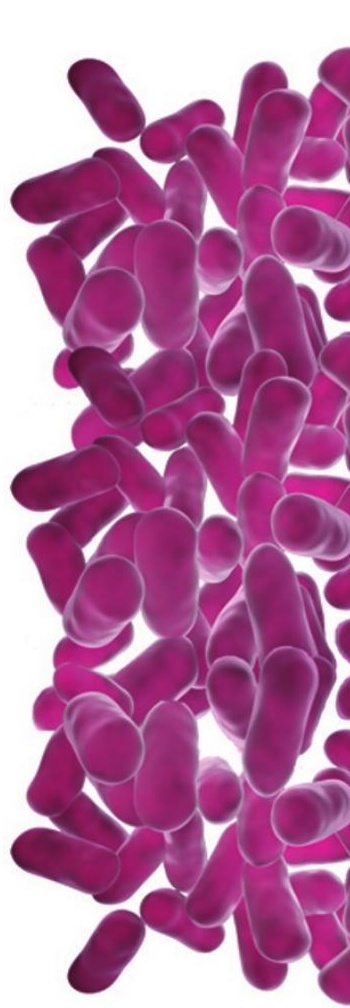

Original articles Szajewska, H. et al. Meta-analysis: the effects of Lactobacillus rhamnosus GG supplementation for the prevention of health-care associated diarrhoea in children. Aliment. Pharmacol. Ther. doi:10.1111/j.13652036.2011.04837.x | Vandenplas, Y. et al. Randomised clinical trial: the synbiotic food supplement Probiotical vs. placebo for acute gastroenteritis in children. Aliment Pharmacol. Ther. 34, 862-867 (2011) 